\title{
Quantum Zeno tomography
}

\author{
P. Facchi, ${ }^{1,2}$ Z. Hradil,${ }^{3}$ G. Krenn, ${ }^{1}$ S. Pascazio, ${ }^{1,2}$ and J. Řeháček ${ }^{3}$ \\ ${ }^{1}$ Dipartimento di Fisica, Università di Bari I-70126 Bari, Italy \\ ${ }^{2}$ Istituto Nazionale di Fisica Nucleare, Sezione di Bari, I-70126 Bari, Italy \\ ${ }^{3}$ Department of Optics, Palacký University, 17. listopadu 50, 772 00 Olomouc, Czech Republic
}

(Dated: November 18, 2018)

\begin{abstract}
We show that the resolution "per absorbed particle" of standard absorption tomography can be outperformed by a simple interferometric setup, provided that the different levels of "gray" in the sample are not uniformly distributed. The technique hinges upon the quantum Zeno effect and has been tested in numerical simulations. The scheme we propose could be implemented in experiments with UV-light, neutrons or X-rays.

PACS numbers: 03.65.Xp
\end{abstract}

\section{INTRODUCTION}

Absorption tomography is an important experimental technique revealing the internal structure of material bodies. By measuring the attenuation of a beam of particles passing through a sample one infers the absorption coefficient (density) of the sample in the beam section. The possibility of distinguishing two slightly different densities of the material is often of vital importance. Under otherwise ideal conditions the shot noise associated with the discrete character of the illuminating beam sets an upper limit to the resolution of absorption tomography: for instance, the shadow cast by a brain tumor might become totally lost in the noisy data. One possibility to overcome the fluctuations is to increase the intensity of the beam. However, in many situations, like in medicine for example, the intensity of the illuminating beam cannot be made arbitrarily high due to the damage provoked by the absorbed radiation.

A significant step towards an "absorption-free tomography" came from quantum theory. It was demonstrated, both theoretically [1, 2] and experimentally [3], that totally transmitting and absorbing bodies can be distinguished without absorbing any particles, by using an interferometric setup. This idea is in fact a clever implementation of the quantum Zeno effect [4] and hinges upon the notion of "interaction-free" measurement [5]. A classical measuring apparatus (here the black sample), placed in one arm of the interferometer, projects the illuminating particle into the other arm, destroying interference, freezing the evolution and forcing the particle to exit through a different channel from that it would have chosen had both arms been transparent (white sample).

In practical applications, however, samples are normally neither black nor white: they are gray. In this paper we endeavor to understand whether application of the quantum Zeno effect, which turns out to be ideal for discriminating black and white, might be advantageous also for the more practical task of discriminating two gray bodies with different transmission coefficients. More specifically, we ask: is it possible by quantum Zeno effect to reduce the number of absorbed particles while preserving the resolution? We show that this is indeed possible. Closely related questions have been recently investigated by other authors [6, 7]. Our conclusions are somewhat optimistic: we show that standard absorption tomography can be outperformed by a Zeno setup, provided that the frequency of occurrences of the different levels of "gray" in the sample is not uniform. More to this, the Zeno setup, unlike the standard one, is endowed with two detection channels: as we shall see, this feature, if properly exploited, leads to even better performances in the Zeno case.

\section{QUANTUM ZENO EFFECT IN A MACH-ZEHNDER INTERFEROMETER}

We introduce notation and sketch the fundamental features of the quantum Zeno effect. We consider the MachZehnder interferometric (MZI) scheme with feedback displayed in Fig. 1h. A semitransparent object, whose transmission amplitude is $\tau$ (assumed real for simplicity) is placed in the lower arm of the interferometer. The particle is initially injected from the left, crosses the interferometer $L$ times and is finally detected by one of two detectors. The two semitransparent mirrors $\mathrm{M}$ are identical and their amplitude transmission and reflection coefficients are

$$
c \equiv \cos \theta_{L}, \quad s \equiv \sin \theta_{L} \quad\left(\theta_{L}=\pi / 4 L\right),
$$

respectively. Notice that both coefficients depend on $L$, the number of "loops" in the MZI.

The incoming state of the particle (coming from the source at initial time) is

$$
\mid \text { in }\rangle=\left(\begin{array}{l}
1 \\
0
\end{array}\right)
$$

and we call "Zeno" and "orthogonal" channels the extraordinary $\left(\begin{array}{l}1 \\ 0\end{array}\right)$ and ordinary $\left(\begin{array}{l}0 \\ 1\end{array}\right)$ channels of the MZI, respectively. The total effect of the interferometer is

$$
V_{\tau}=B A_{\tau} B, \quad B=\left(\begin{array}{cc}
c & -s \\
s & c
\end{array}\right), \quad A_{\tau}=\left(\begin{array}{ll}
1 & 0 \\
0 & \tau
\end{array}\right) .
$$


(a)

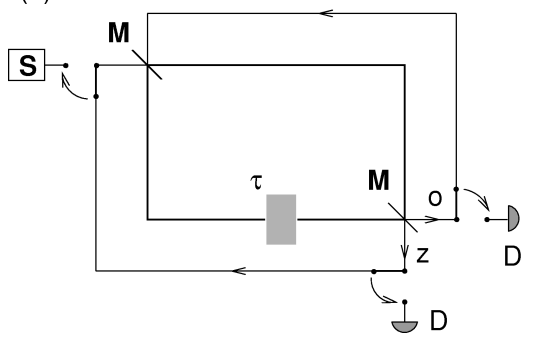

(b)

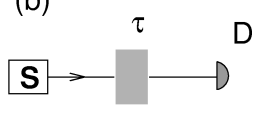

FIG. 1: a) Scheme of the Zeno interferometric setup. b) Standard transmission experiment. $\mathrm{S}$ - source; $\mathrm{M}$ - semitransparent mirror; o - orthogonal channel; z -Zeno channel; $\mathrm{D}$ - detector.

In general,

$$
B=\exp \left(-i \theta_{L} \sigma_{2}\right), \quad B B^{\dagger}=B^{\dagger} B=1,
$$

where $\sigma_{2}$ is the second Pauli matrix, while $A_{\tau}$ is not unitary (if $\tau<1$ there is a probability loss). The final state, after the particle has gone through $L$ loops, reads

$$
\left.\left.\mid \text { out }\rangle=V_{\tau}^{L} \mid \text { in }\right\rangle=\left(B A_{\tau} B\right)^{L} \mid \text { in }\right\rangle .
$$

\section{A. White sample}

The choice of the angle $\theta_{L}$ in (1) is motivated by our requirement that if $\tau=1$ ("white" sample, i.e. no semitransparent object in the MZI) the particle ends up in the "orthogonal" channel:

$$
V_{\tau=1}^{L}=B^{2 L}=e^{-i 2 L \theta_{L} \sigma_{2}}=e^{-i \pi \sigma_{2} / 2}=-i \sigma_{2},
$$

so that

$$
\left.\mid \text { out }\rangle=V_{\tau=1}^{L} \mid \text { in }\right\rangle=\left(\begin{array}{l}
0 \\
1
\end{array}\right) .
$$

This is easy to understand: each loop "rotates" the particle's state by $2 \theta_{L}=\pi / 2 L$ and after $L$ loops the final state is "orthogonal" to the initial one (2).

\section{B. Black sample}

Let us now look at the case $\tau=0$, corresponding to a completely opaque ("black") object in the MZI. We obtain

$$
\begin{aligned}
V_{\tau=0}^{L} & =B\left(A_{0} B^{2}\right)^{L} B^{-1} \\
& =B \cos ^{L} 2 \theta_{L}\left(\begin{array}{cc}
1 & -\tan 2 \theta_{L} \\
0 & 0
\end{array}\right) B^{-1} \\
& \stackrel{L \rightarrow \infty}{\longrightarrow}\left(\begin{array}{ll}
1 & 0 \\
0 & 0
\end{array}\right) \equiv \mathcal{V}_{\tau=0} .
\end{aligned}
$$

This yields QZE:

$$
\left.\left.\mid \text { out }\rangle=\mathcal{V}_{\tau=0} \mid \text { in }\right\rangle=\mid \text { in }\right\rangle=\left(\begin{array}{l}
1 \\
0
\end{array}\right) .
$$

In the infinite $L$ limit the initial state is "frozen" and the particle ends up in the Zeno channel.

\section{Gray sample}

What happens if $0<\tau<1$ ? We easily get

$$
V_{\tau}=\left(\begin{array}{cc}
(1+\tau) c^{2}-\tau & -s c(1+\tau) \\
s c(1+\tau) & \tau-(1+\tau) s^{2}
\end{array}\right) .
$$

The computation of $V_{\tau}^{L}$ is straightforward but lengthy and yields a final expression which is elementary but complicated. However, we are mainly interested in the large- $L$ limit, that for

$$
\tau<\tau_{L}^{Z} \equiv(1-\sin \pi / 2 L) /(1+\sin \pi / 2 L)
$$

reads 13 ]

$$
V_{\tau}^{L}=\left(\begin{array}{cc}
1-\frac{\pi^{2}}{8 L} \frac{1+\tau}{1-\tau} & \mathrm{O}\left(L^{-1}\right) \\
\mathrm{O}\left(L^{-1}\right) & \tau^{L}\left[1+\mathrm{O}\left(L^{-1}\right)\right]
\end{array}\right)+\mathrm{O}\left(L^{-2}\right) .
$$

This is an interesting result: indeed

$$
\mathcal{V}_{\tau} \equiv \lim _{L \rightarrow \infty} V_{\tau}^{L}=\left(\begin{array}{ll}
1 & 0 \\
0 & 0
\end{array}\right), \quad 0 \leq \tau<1
$$

analogously to (8). This shows that even for a semitransparent object, with transmission coefficient $\tau \neq 1$, a bona fide QZE takes place and the particle ends up in the Zeno channel with probability one:

$$
\left.\left.\mid \text { out }\rangle=\mathcal{V}_{\tau} \mid \text { in }\right\rangle=\mid \text { in }\right\rangle, \quad \tau \neq 1 .
$$

\section{DISTINGUISHING DIFFERENT SHADES OF GRAY}

A question arises [7]: is it possible to distinguish different values of $\tau$ (different "shades" or "levels" of gray) by the technique outlined above? This is not a simple task, for after a large number of loops $L$ the particle ends up in the orthogonal channel only if $\tau=1$ [see Eq. (7)]; by contrast, for any value of $\tau \neq 1$, the particle ends up in the Zeno channel [see (14)] irrespectively of the particular value of $\tau$. However, the asymptotic correction in the $(1,1)$ element of $V_{\tau}^{L}$ in (12) is $\tau$ dependent: the details of the convergence to the limit (13)-(14) depend on the grayness of the sample. By exploiting this feature, we shall now show that it is indeed possible to resolve different gray levels by QZE, within a given statistical accuracy.

We start by observing that if one performs a standard transmission experiment, by shining a particle beam on a semitransparent object in order to measure the transmission coefficient $\tau$, see Fig. \b, the detection and absorption probabilities read

$$
p_{d}^{\prime}(\tau)=\tau^{2}, \quad p_{a}^{\prime}(\tau)=1-\tau^{2} .
$$


The statistics is binomial.

On the other hand, if one uses the Zeno configuration sketched in Fig. 11a, the final state of the particle after $L$ loops in the MZI is, from (12),

$$
V_{\tau}^{L}\left(\begin{array}{l}
1 \\
0
\end{array}\right)=\left(\begin{array}{l}
u_{z} \\
u_{o}
\end{array}\right)=\left(\begin{array}{c}
1-\frac{\pi^{2}}{8 L} \frac{1+\tau}{1-\tau}+\mathrm{O}\left(L^{-2}\right) \\
\mathrm{O}\left(L^{-1}\right)
\end{array}\right)
$$

where $u_{z}$ and $u_{o}$ are the amplitudes in the Zeno and orthogonal channels, respectively. Both these quantities are real. Let $(0 \leq \tau<1)$

$$
\begin{aligned}
& p_{z}(\tau)=u_{z}^{2}=1-\frac{\pi^{2}}{4 L} \frac{1+\tau}{1-\tau}+\mathrm{O}\left(L^{-2}\right) \\
& p_{o}(\tau)=u_{o}^{2}=\mathrm{O}\left(L^{-2}\right) \\
& p_{a}(\tau)=1-p_{z}(\tau)-p_{o}(\tau)=\frac{\pi^{2}}{4 L} \frac{1+\tau}{1-\tau}+\mathrm{O}\left(L^{-2}\right)
\end{aligned}
$$

be the probabilities that the particle is detected in the Zeno, orthogonal channel or is absorbed by the semitransparent object, respectively. We assume that a fixed number of particles $N$ is sent in the MZI during an experimental run. In this situation the distribution of particles in the Zeno, orthogonal or absorption channels follows a trinomial statistics with probabilities (17).

It seems natural to think that, since by increasing the number of loops $L, p_{o}$ vanishes much faster than $p_{a}$, for large $L$ the distribution is practically binomial with $p_{a}+$ $p_{z} \approx 1$ [14]. However, as we shall see, the presence of a small trinomial component, $p_{o}$, will play an important role, enabling the Zeno method to perform much better than the standard one.

\section{AN INTRINSIC LIMIT FOR BINOMIAL STATISTICS: THE CRAMÉR-RAO BOUND}

We are now ready to discuss the possibility of a "Zeno tomography." The goal is to get information about the distribution of the absorption coefficient in the sample, absorbing as few particles as possible. We will accomplish this in two steps. First, using estimation theory, we show that if one limits one's attention to binomial statistics, the Zeno estimation of any level of gray of one pixel (i.e. $\tau$ continuously distributed between 0 and 1) cannot perform better than the standard method. At best, both methods are equivalent. This is bad news. However, the very proof of the above-mentioned statement will show that there are two ways out: first, we will see that Zeno performs better when one wants to distinguish two levels of gray that are not equally populated in the sample (this requires some prior knowledge about the distribution of grays in the sample). This is good news, for it enables one to find a method that in some cases works better than the standard one. Second, one is led to think that the introduction and exploitation of a trinomial statistics can enable the Zeno method to perform better.

Let us start from the estimation of any level of gray. In this case one tries to estimate $\tau^{2}$ from the counted number of particles, absorbing as few particles as possible for the requested precision. To perform this task in an optimal way one should find optimal estimators for each scheme. A lower bound on the variance of an unbiased estimator $\hat{T}$ of the parameter $T$ (here $T=\tau^{2}$ ) is the Cramér-Rao lower bound (CRLB) [8],

$$
(\Delta \hat{T})^{2} \geq \frac{1}{F} \equiv\left\langle\left[\frac{\partial}{\partial T} \ln p(n \mid T)\right]^{2}\right\rangle^{-1}
$$

where $F$ is the Fisher information, $p(n \mid T)$ the probability of observing $n$ particles conditioned by the value $T$ of the unknown parameter and $\langle\ldots\rangle$ denotes ensemble average with respect to $n$. The probability $p$ is binomially distributed in both the standard case and the Zeno case, which yields

$$
\begin{aligned}
\left(\Delta \hat{T}_{\mathrm{st}}\right)^{2} & \geq \frac{\tau^{2}\left(1-\tau^{2}\right)}{N} \\
\left(\Delta \hat{T}_{\mathrm{Ze}}\right)^{2} & \geq \frac{4 \tau^{2}(1-\tau)^{3}(1+\tau) L}{\pi^{2} N}
\end{aligned}
$$

for standard and Zeno tomography, respectively, $N$ being the (fixed) number of input particles in both cases. Expressing the above inequalities (19) in terms of the number of absorbed particles $N_{a}^{(\mathrm{st})}=N p_{a}^{\prime}$ and $N_{a}^{(\mathrm{Ze})}=N p_{a}$, they both reduce to the same bound

$$
\Delta \hat{T}_{\mathrm{st}, \mathrm{Ze}}^{\mathrm{opt}} \geq \frac{\tau\left(1-\tau^{2}\right)}{\sqrt{N_{a}^{(\mathrm{st}),(\mathrm{Ze})}}}
$$

showing that the CRLB's for standard and Zeno tomography are the same, given the number of absorbed particles. Further, it is trivial to show that the unbiased estimator given by the relative frequency of transmitted particles, $\hat{T}_{\mathrm{st}}=n_{t} / N$, saturates the CRLB (20). Hence, if one neglects the output of the ordinary channel $p_{o}$ and considers the statistics (17) practically binomial, the Zeno estimation can be at most as good as the standard one: it cannot be better.

\section{TWO STATISTICAL PROTOCOLS}

\section{A. Binomial (single-channel) protocol}

In spite of the conclusions of Sec. IV] we will now construct a protocol and show that by QZE one can achieve a resolution that is superior to the "ordinary" resolution obtained in a standard transmission experiment. Notice that $p_{a}(\tau)$ in (17), unlike $p_{a}^{\prime}(\tau)$ in (15), is an increasing function of $\tau$. Therefore, with respect to absorbed particles, the Zeno tomographic image (for sufficiently large $L)$ yields a kind of negative of the standard absorption tomographic image. This can be given a rather intuitive explanation: indeed, the absorption probability in (17) reduces to the same form as the standard one (15), i.e.

$$
p_{a}(\tau)=1-\left(\tau_{\mathrm{eff}}^{\mathrm{Ze}}\right)^{2},
$$


by introducing an "effective" transmission coefficient

$$
\tau_{\text {eff }}^{\mathrm{Ze}}=\sqrt{1-p_{a}} .
$$

For example, if we take $\tau_{1}=0.98, \tau_{2}=0.99$ and choose $L=12000$, then, according to Eq. (17), we get $\tau_{\mathrm{eff} 1}^{\mathrm{Ze}} \approx 0.99$ and $\tau_{\mathrm{eff} 2}^{\mathrm{Ze}} \approx 0.98$. The two gray levels are interchanged by the Zeno apparatus. If most of the sample has transmission coefficient $\tau_{2}$ the absorbed energy is reduced by using the Zeno setup.

A more precise comparison of the performances of the Zeno and standard techniques can be given in the framework of decision theory. For simplicity let us focus on distinguishing only two gray levels $\tau_{1}$ and $\tau_{2}\left(\tau_{1}<\tau_{2}\right)$ corresponding to hypotheses $H_{1}$ and $H_{2}$ that occur in the sample with frequencies

$$
P_{0}\left(H_{1}\right)=\alpha, \quad P_{0}\left(H_{2}\right)=1-\alpha .
$$

With this simplification we lose no generality since the tomography with $M$ gray levels can always be split into a sequence of pairwise decisions between two adjacent gray levels.

We will proceed in two steps. First we will assume that in the Zeno configuration of Fig. 17 all output particles are collected at a single detector. In other words, the Zeno and orthogonal channels are considered as a single output and the statistics (17) is binomial $\left(p_{z}+p_{o}, p_{a}\right)$. Each particle is then either absorbed or transmitted (and detected) by the Zeno apparatus. Obviously, by merging the two output channels together, some information about the sample is wasted. We know, however, that this strategy will be optimal if the number of loops $L$ is very large. Then there are almost no particles exiting via the ordinary channel $n_{o} \approx 0$ which can then be safely ignored. A better and more general strategy will be studied later.

Since both experiments obey the same (binomial) statistics we use the notation of the Zeno experiment. The analysis of the standard experiment is similar. If no distinction is made between the Zeno and ordinary channels, the decision is based on the number of absorbed particles $n_{a}$. If $n_{a}$ is smaller than or equal to a decision level $n_{a}^{d}$, then $H_{1}$ is chosen; otherwise $H_{2}$ is chosen. The probability of making an error in identifying the gray level of a given pixel is

$$
P_{e}=\alpha P\left(H_{2} \mid H_{1}\right)+(1-\alpha) P\left(H_{1} \mid H_{2}\right),
$$

where

$$
P\left(H_{1} \mid H_{2}\right)=\sum_{n_{a} \leq n_{a}^{d}} p\left(n_{a} \mid H_{2}\right)
$$

is the probability of choosing $H_{1}$ when $H_{2}$ is true and

$$
p\left(n_{a} \mid H_{2}\right)=\left(\begin{array}{c}
N \\
n_{a}
\end{array}\right) p_{a}\left(\tau_{2}\right)^{n_{a}}\left[1-p_{a}\left(\tau_{2}\right)\right]^{N-n_{a}}
$$

is the binomial probability of absorbing $n_{a}$ particles when $H_{2}$ is true. [For $P\left(H_{2} \mid H_{1}\right)$ the summation is over $n_{a}>$ $\left.n_{a}^{d}\right]$. An optimal protocol is given by determining $n_{a}^{d}$ that minimizes the error (24).

Alternatively, one defines the likelihood ratio [9]

$$
R=\frac{\mathcal{L}\left(\tau_{1} \mid N, n_{a}, \alpha\right)}{\mathcal{L}\left(\tau_{2} \mid N, n_{a}, 1-\alpha\right)}
$$

where

$$
\begin{aligned}
\mathcal{L}\left(\tau_{i} \mid N, n_{a}, \alpha\right) & =P_{0}\left(H_{i}\right) p\left(n_{a} \mid H_{i}\right) \\
& =\alpha\left(\begin{array}{c}
N \\
n_{a}
\end{array}\right) p_{a}\left(\tau_{i}\right)^{n_{a}}\left[1-p_{a}\left(\tau_{i}\right)\right]^{N-n_{a}}
\end{aligned}
$$

represents the likelihood of hypothesis $H_{i}(i=1,2)$. The optimum decision level $n_{a}^{d}$ is determined by solving for equal likelihoods [15]

$$
R=1
$$

In both cases one gets

$$
n_{a}^{d}=\frac{\log \left[\frac{1-\alpha}{\alpha}\right]-N \log \left[\frac{1-p_{a}\left(\tau_{1}\right)}{1-p_{a}\left(\tau_{2}\right)}\right]}{\log \left[\frac{p_{a}\left(\tau_{1}\right)}{p_{a}\left(\tau_{2}\right)}\right]-\log \left[\frac{1-p_{a}\left(\tau_{1}\right)}{1-p_{a}\left(\tau_{2}\right)}\right]}
$$

and, substituting in (24),

$$
\begin{aligned}
P_{e}= & \alpha\left\{1-B_{I}\left[N-\tilde{n}_{a}^{d}, 1+\tilde{n}_{a}^{d} ; 1-p_{a}\left(\tau_{1}\right)\right]\right\} \\
& +(1-\alpha) B_{I}\left[N-\tilde{n}_{a}^{d}, 1+\tilde{n}_{a}^{d} ; 1-p_{a}\left(\tau_{2}\right)\right],
\end{aligned}
$$

where $B_{I}(a, b ; z)$ is the regularized incomplete Beta function [10] and $\tilde{n}_{a}^{d}$ is the greatest integer less than or equal to $n_{a}^{d}$. The mean number of absorbed particles is

$$
N_{a}^{(\mathrm{Ze})}=N\left[\alpha p_{a}\left(\tau_{1}\right)+(1-\alpha) p_{a}\left(\tau_{2}\right)\right] .
$$

By plugging Eqs. (30) and (32) in Eq. (31), the average probability of error (31) can be expressed as a function of $\alpha, \tau \equiv \tau_{1}, d \tau=\tau_{2}-\tau_{1}$ and $N_{a}^{(\mathrm{Ze})}$. The probability of error for the standard setup is obtained in a completely analogous way.

The performances of the Zeno and standard methods are compared in Fig. 2 First, the (exact) $L$ th power of the matrix $V_{\tau}$ in (10) is evaluated, then for a given error rate $P_{e}$, the number of absorbed particles is calculated by solving numerically Eq. (31). Their ratio is shown as a function of $\alpha$ for a few values of the transmission coefficient $\tau$. Notice that the exposition of the sample can be significantly reduced if the distribution of gray levels in the sample is not uniform. For instance, a reduction factor of 2.5 is obtained when the sample consists of $97 \%$ of dense material and $3 \%$ of the less absorbing one, $\alpha=0.97$. Such parameters are typical for structural analyses: a small structural defect (crack) inside a thin sample would typically show small contrast $(\delta \tau \ll 1)$ with the surrounding almost transparent $(\tau \approx 1)$ material, while its area would be small compared to the area of the sample $(\alpha \approx 1)$. 


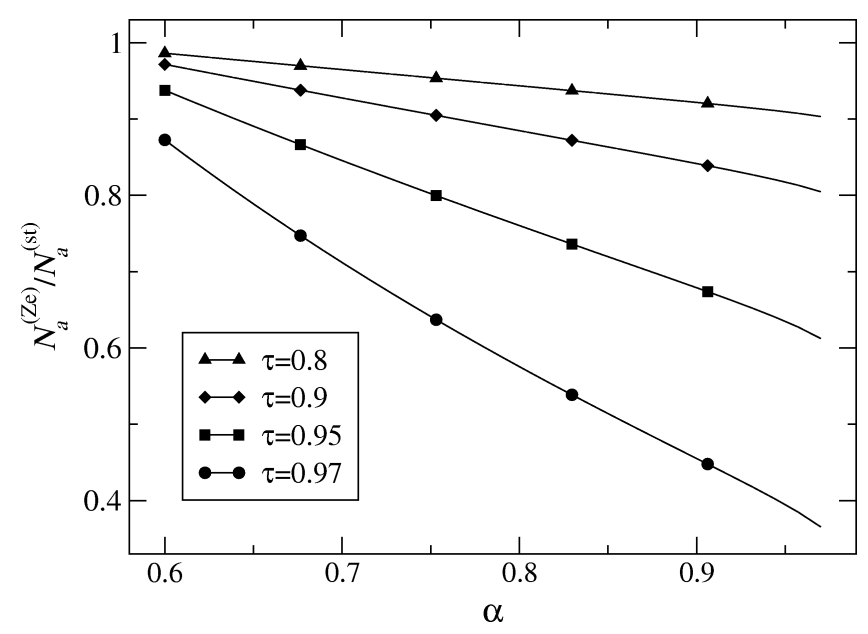

FIG. 2: Ratio of the number of absorbed particles in the Zeno $\left(N_{a}^{(\mathrm{Ze})}\right)$ and standard $\left(N_{a}^{(\mathrm{st})}\right)$ setup. The smaller the ratio in the graph, the less irradiation in the Zeno apparatus (for the same resolution). $P_{e}=0.5 \% ; d \tau=0.02$; $\tau=\{0.8,0.9,0.95,0.97\} ; L=2000$.

\section{B. Trinomial (two-channel) protocol}

The binomial decision strategy outlined in the previous subsection is not the optimum one. Unavoidable losses and other imperfections of real experimental devices set a strict limit on the maximum number of loops that can be achieved in a laboratory. In such case the ordinary channel can no longer be ignored. The data consist then of the two component vector $\left(n_{z}, n_{o}\right)$ of the numbers of particles counted in the Zeno and ordinary output channels. The decision will be based on both these numbers.

The decision levels are readily obtained from the equal likelihood criterion

$$
R=\frac{\mathcal{L}\left(\tau_{1} \mid N, n_{z}, n_{o}, \alpha\right)}{\mathcal{L}\left(\tau_{2} \mid N, n_{z}, n_{o}, 1-\alpha\right)}=1,
$$

where

$$
\mathcal{L}\left(\tau \mid N, n_{z}, n_{o}, \alpha\right)=\frac{\alpha N !}{n_{z} ! n_{o} ! n_{a} !} p_{z}(\tau)^{n_{z}} p_{o}(\tau)^{n_{o}} p_{a}(\tau)^{n_{a}} .
$$

This equation is to be solved for the decision vector $\left(n_{z}^{d}, n_{o}^{d}\right)$. Equation (33) is just one condition for the two unknowns $n_{z}$ and $n_{o}$, so there exists a one-parametric family of solutions. By plugging (34) into (33) one easily finds

$$
n_{z}^{d}-a\left(\tau_{1}, \tau_{2}\right) n_{o}^{d}=b\left(\tau_{1}, \tau_{2}, \alpha\right)
$$

where the coefficients $a$ and $b$ read

$$
\begin{aligned}
& a=\frac{\log \left[\frac{p_{o}\left(\tau_{1}\right) p_{a}\left(\tau_{2}\right)}{p_{o}\left(\tau_{2}\right) p_{a}\left(\tau_{1}\right)}\right]}{\log \left[\frac{p_{z}\left(\tau_{2}\right) p_{a}\left(\tau_{1}\right)}{p_{z}\left(\tau_{1}\right) p_{a}\left(\tau_{2}\right)}\right]}, \\
& b=\frac{N \log \left[\frac{p_{a}\left(\tau_{1}\right)}{p_{a}\left(\tau_{2}\right)}\right]+\log \left[\frac{\alpha}{1-\alpha}\right]}{\log \left[\frac{p_{z}\left(\tau_{2}\right) p_{a}\left(\tau_{1}\right)}{p_{z}\left(\tau_{1}\right) p_{a}\left(\tau_{2}\right)}\right]} .
\end{aligned}
$$

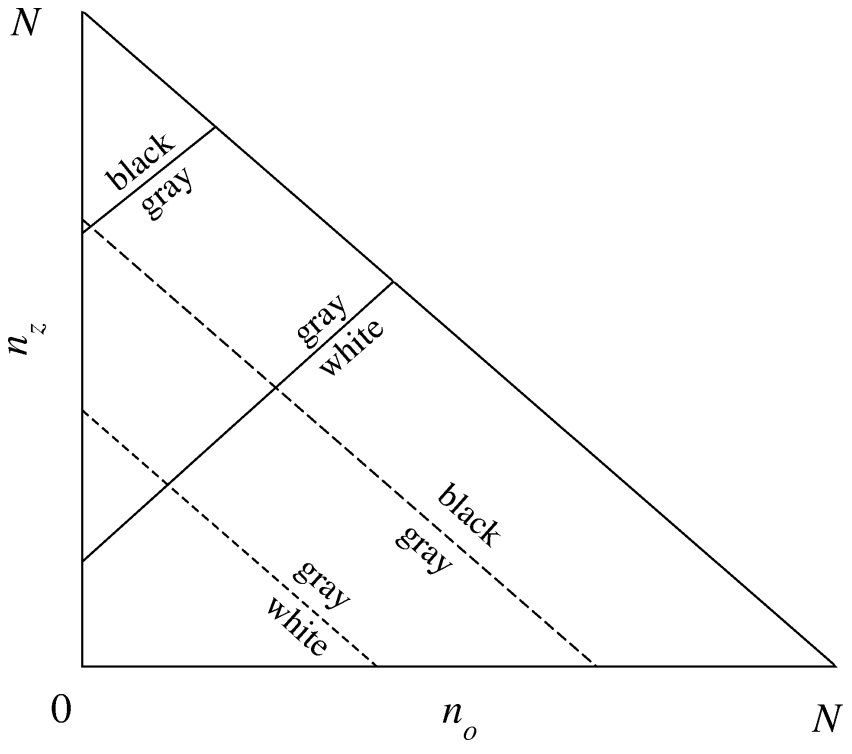

FIG. 3: Typical decision levels of the binomial (dashed lines) and trinomial (solid lines) decision strategies when $a \approx 1$. This figure corresponds to the simulation shown in the last row of Fig. 5: where $M=3$, the triangle is divided into $M=3$ regions and the three gray shades are labelled as white, gray and black, respectively.

In a two-dimensional representation each possible experimental outcome $\left(n_{z}, n_{o}\right)$ is represented by a point lying inside the triangle $\left\{0 \leq n_{o}+n_{z} \leq N\right\}$ shown in Fig. 3. Equation (35) divides this triangle into two regions. All experimental outcomes that fall within the same region issue the same decision. In the general case of $M$ different gray levels there are $M-1$ equations (35) defining $M-1$ in general non-parallel lines dividing the square into $M$ strip-like regions. This is shown in Fig. 3 for $M=3$.

An interesting situation arises when the coefficient $a$ in (35) becomes close to unity. In that case, the decision level is the line $n_{z}^{d}-n_{o}^{d}=$ const. Let us recall that the decision levels of the binomial decision strategy discussed in the previous subsection were $n_{a}^{d}=$ const. (see (30) ), or, equivalently, $n_{z}^{d}+n_{o}^{d}=$ const. Hence if $L, \tau_{1}$ and $\tau_{2}$ are such that $a \approx 1$, the decision levels of the binomial and trinomial decision strategies are orthogonal to each other. This is shown in Fig. 3] Under such conditions one can expect further gain in the precision of the Zeno apparatus as compared to standard absorption tomography. This regime was chosen for our computer simulations of the following section.

Notice that the steepness of the decision lines (35) depends only on the absorption of the corresponding adjacent gray levels. It depends neither on their frequencies, nor on the total number of incident particles.

Finally, let us discuss the limit $L \rightarrow \infty$. When the number of loops $L$ increases, $p_{o} \rightarrow 0$ much faster than $p_{a}$ [see (17)] and one is allowed to put $n_{o}^{d}=0$ and $n_{z}^{d}=$ $N-n_{a}^{d}$ in Eq. 35), which reduces to

$$
n_{a}^{d}=N-b\left(\tau_{1}, \tau_{2}, \alpha\right) .
$$




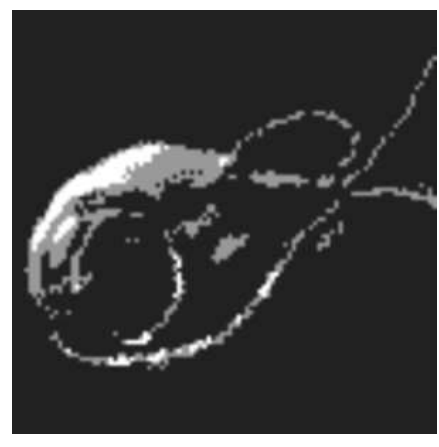

FIG. 4: The object to be reconstructed: a cell of Giardia lamblia, one of the most primitive eukaryotes. The original picture has been reduced for simplicity to three levels of gray: white, gray and black, occurring with frequencies $\alpha_{w}=0.02$, $\alpha_{g}=0.07$ and $\alpha_{b}=0.93$ respectively.

By substituting $p_{z}(\tau)=1-p_{a}(\tau)$ in (37)-(38), one reobtains the binomial condition (30): the binomial strategy becomes optimal in this limit.

\section{SIMULATIONS}

We have seen that the Zeno technique can reduce the level of absorption without losing resolution (compared to the standard technique). (Alternatively, the Zeno setup can yield an improved resolution, while keeping the absorption at the same level of the standard setup.) The object in Fig. 团is a cell of giardia lamblia, a protist, one of the most primitive eukaryotes. Giardia has been called a "missing link" in the evolution of eukaryotic cells from prokaryotic cells. The number of gray levels in the figure has been reduced to three to make the analysis simpler: white, gray and black, $\tau_{w}=0.99, \tau_{g}=0.96, \tau_{b}=0.8$, occurring with frequencies $\alpha_{w}=0.02, \alpha_{g}=0.07$, and $\alpha_{b}=0.93$ respectively. Figure 5 shows the results of a numerical simulation, performed with the standard and Zeno methods, the latter for $L=10$ and $L=165$, for different numbers of absorbed particles $N_{a}$. In each frame the standard and the two Zeno reconstructions are compared, together with the pixels that have been misinterpreted. Figure 5 confirms the expectation based on the asymptotic formulas (17): in general, provided that the object contains a small fraction of more transparent pixels and a larger fraction of more absorbing material, the Zeno setup yields a better resolution for a given irradiation. Clearly, a significant improvement with respect to standard absorption tomography is achieved for as few as $L=10$ loops. The improvement is very large for $L=165$.

The number of absorbed particles increases from (a) to (d) in Fig. 5 Observe that in (a) the standard reconstruction fails completely, while the outline and basic shape of the object can be recognized already in the Zeno reconstruction with $L=10$. In (c) the Zeno reconstructions are quite good, while standard tomography does not detect white pixels in the object. When the intensity of the illuminating beam is increased further, in frame (d), all the reconstructed images become visually hard to tell from the sample, but the error rates of the Zeno apparatuses are still much better (by a factor three or more), as shown by the number of misinterpreted pixels.

It is worth commenting on the distribution of misinterpreted pixels. Clearly, in all the cases analyzed, it is not uniform. In general, when the distribution of gray levels in the sample is not uniform, any reconstruction technique tends to perform better in the "background," while making more mistakes in the region where the "structure" is present. The improvement due to the Zeno method becomes apparent if one looks in particular at Figures 5 (b) and (c): in these cases, interestingly, the standard method yields more mistakes in the background; this is an unpleasant feature, if one is interested in detecting small irregular structures in a more or less uniform background. The features of the distribution of misinterpreted pixels require more careful study and their comprehension might lead to additional ideas.

Any increase in the number of loops $L$ in the interferometer makes the difference between standard and Zeno tomography even bigger. Clearly, this is more demanding in terms of experimental realization.

\section{CONCLUSIONS}

We have shown that a quantum Zeno tomography is possible and performs better than standard tomography if a given prior knowledge about the distribution of grays in the sample is available. This is a common situation in radiography, where one is often interested in detecting a small structure in a uniform background, like for instance in the analysis of small structural defects.

In our numerical simulations we have illustrated some situations in which the resolution is improved by the Zeno method, for a given number of absorbed particles. Alternatively, for a given resolution, the Zeno method performs better, absorbing less particles. This can be interesting in applications, for instance if one wants to limit the damage provoked by the absorption of radiation without losing in resolution.

It is obvious from Fig. 2 that an even larger improvement is possible for almost transparent samples, provided that $\alpha$ is close to unity. This means that there is no fundamental limit on the improvement that can be achieved over the standard setup: in other words, there is no "optimal" configuration.

There are additional issues that deserve careful study. For instance, the effects due to a Poissonian beam (total number of incoming particles $N$ not fixed) and a complex transmission coefficient $\tau$.

Let us also comment on experimental feasibility. Figure 5 shows that an experimental test of the Zeno tomographic technique should not be as difficult as one might think: simulations have been performed for as few as $L=10$ loops in the interferometer, giving better results 


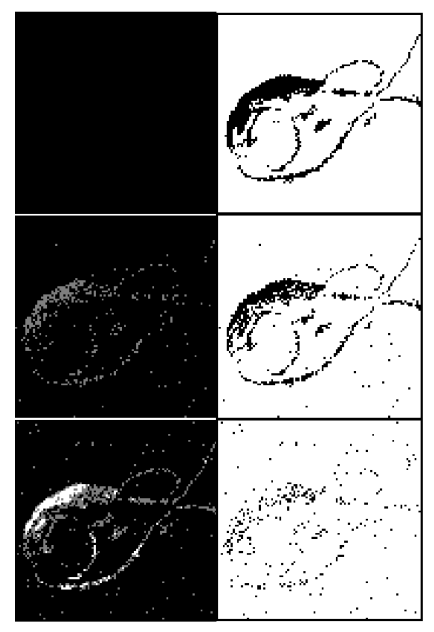

(a)

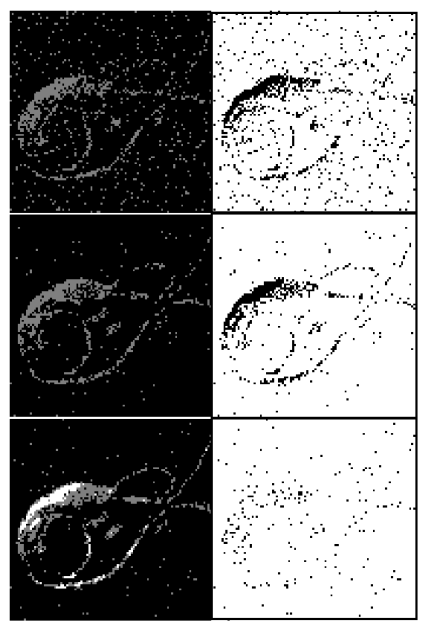

(b)

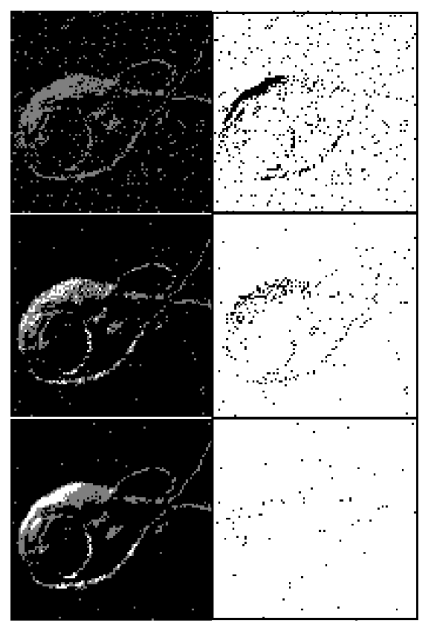

(c)

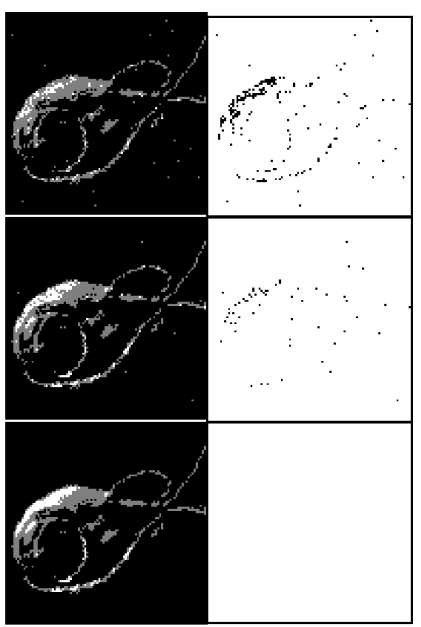

(d)

FIG. 5: Comparison of standard and Zeno tomographic techniques. In each frame: top left=reconstruction by standard technique; top right=misinterpreted pixels by the standard technique; center left=reconstruction by Zeno technique with $L=10$; center right=misinterpreted pixels by the Zeno technique with $L=10$; bottom left=reconstruction by Zeno technique with $L=165$; bottom right=misinterpreted pixels by the Zeno technique with $L=165$. The mean number of absorbed particles per pixel (irradiation) is $N_{a}=1.7,2.3,4$ and 13 for frames (a), (b), (c) and (d) respectively. The total number of particles $N$ (total energy) scales approximately like $3 N_{a}, 1.8 N_{a}$, and $6.5 N_{a}$ for top, center and bottom reconstructions, respectively. We used $\tau_{w}=0.99, \tau_{g}=0.96$, and $\tau_{b}=0.8$. The sample consists of $10,000(=100 \times 100)$ pixels, where white, gray and black occur with frequencies $\alpha_{w}=0.02, \alpha_{g}=0.07$ and $\alpha_{b}=0.93$, respectively. The number of misinterpreted pixels are (top to bottom): (a) $968,786,315$; (b) $942,596,212$; (c) $717,382,68$; (d) 205, 69, 0 .

than the standard method. It is reasonable to think that a Zeno setup with a much larger number of loops can be built for UV light (highly absorbed by some biological samples). Also, by changing the light wavelength, one could efficiently "observe" different regions of the sample (or slightly different samples). Moreover, the experimental configuration we have proposed (photons in a MZI, like in Fig. 1) is certainly not the only conceivable one. Phase imaging and tomography have been demonstrated for both X-rays and neutrons [1]. More to this, Rauch and collaborators, with the VESTA apparatus [12], have been able to keep neutrons in a one-meter long perfect crystal storage system ("resonator") for a few seconds, so that the neutrons bounce back and forth between two mirrors several thousands times. This would lead us to the full asymptotic $(L \gg 1)$ regime considered in Fig. 2 and last row of Fig. 5 where the Zeno method can perform much better. There is hopefully more to come.

\section{Acknowledgments}

This paper is dedicated to Professor Jan Peřina on the occasion of his 65 th birthday. This work is supported by the TMR-Network of the European Union "Perfect Crystal Neutron Optics" ERB-FMRX-CT96-0057. J. ̌̌. and Z. H. acknowledge support by the project LN00A015.
[1] A. C. Elitzur and L. Vaidman, Found. Phys. 23, 987 (1993).

[2] M. Hafner and J. Summhammer, Phys. Lett. A 235, 563 (1997).

[3] P. Kwiat, H. Weinfurter, T. Herzog, A. Zeilinger, and M. Kasevich, Phys. Rev. Lett. 74, 4763 (1995).

[4] B. Misra and E. C. G. Sudarshan, J. Math. Phys. 18, 756 (1977); S. Pascazio, M. Namiki, G. Badurek, and H. Rauch, Phys. Lett. A 179, 155 (1993). For a review, see H. Nakazato, M. Namiki, and S. Pascazio, Int. J. Mod. Phys. B 10, 247 (1996); D. Home and M. A. B. Whitaker, Ann. Phys. 258, 237 (1997); P. Facchi and S. Pascazio, Progress in Optics, edited by E. Wolf (Elsevier,
Amsterdam, 2001), Vol. 42, Ch. 3, p. 147.

[5] R. H. Dicke, Am. J. Phys. 49, 925 (1981); Found. Phys. 16, 107 (1986).

[6] G. Krenn, J. Summhammer, and K. Svozil, Phys. Rev. A 61, 052102 (2000).

[7] G. Mitchinson and S. Massar, Phys. Rev. A 63, 032105 (2001); S. Massar, G. Mitchison, and S. Pironio Phys. Rev. A 65, 022110 (2002).

[8] C. R. Rao, Bull. Calcutta Math. Soc. 37, 81 (1945); H. Cramér, "Mathematical methods of statistics" (Princeton University Press, 1946).

[9] C. W. Helstrom, "Quantum detection and estimation theory" (Academic Press, New York, 1976). 
[10] M. Abramovitz and I. A. Stegun, "Handbook of mathematical functions" (Dover, New York, 1972), 26.5.1 and 26.5.7.

[11] F. Dubus, U. Bonse, T. Biermann, M. Baron, F. Beckmann, and M. Zawisky, Proceedings SPIE 4503, 359 (2002).

[12] E. Jericha, D. E. Schwab, M. R. Jäkel, C. J. Carlile, and H. Rauch, Physica B 283, 414 (2000); H. Rauch, Physica B 297, 299 (2001).

[13] It is worth stressing that when $\tau>\tau_{L}^{Z}$ the asymptotic expansion has a completely different form and tends to $-i \sigma_{2}$ when $L \rightarrow \infty$. Upon crossing the threshold $\tau=\tau_{L}^{Z}$, the apparatus starts operating in the Zeno regime. We also note that $\tau_{L}^{Z}$ in (11) is bounded from below according to the simple expression $\tau_{L}^{Z}>1-\pi / L$. Alternatively, one can rephrase these conditions in terms of $L$ : the asymptotic expansion (12) is then valid for $L>L_{\tau}^{\mathrm{Z}}=\pi / 2 \arcsin [(1-\tau) /(1+\tau)]$.

[14] More so, since $p_{a}$ is also small in this limit, the detection statistics is almost Poissonian.

[15] The likelihood criterion (27) is also valid for other (non binomial) statistics and can be easily generalized to the case of more than two gray levels. 